\title{
Qualidade de vida, otimismo, enfrentamento, morbidade psicológica e estresse familiar em pacientes com câncer colorrectal em quimioterapia
}

\author{
Ana Sofia Cordeiro Botelho \\ Centro de Saúde de Ponta Delgada \\ Maria da Graça Pereira \\ Universidade do Minho
}

\begin{abstract}
Resumo
Este estudo avaliou as relações entre a qualidade de vida, otimismo, enfrentamento, morbidade psicológica e estresse familiar, os preditores da qualidade de vida e da morbidade psicológica e analisou as diferenças nestas variáveis em função do sexo, do tipo e da duração do tratamento, idade e nível de escolaridade em pacientes com câncer colorretal. Uma amostra de 100 pacientes em tratamento de quimioterapia participou no estudo. Os resultados revelaram que as mulheres, os pacientes diagnosticados nos últimos 12 meses e os que realizaram cirurgia/quimioterapia apresentam maior risco de desenvolver morbidade psicológica. A depressão mostrou ser um preditor da qualidade de vida. A idade e a escolaridade mostraram ser relevantes particularmente ao nível das estratégias de enfrentamento utilizadas e na qualidade de vida. Os resultados enfatizam a necessidade das intervenções focarem a morbidade, otimismo e ter em consideração o gênero, a duração do diagnóstico e o tipo de tratamento, em particular.
\end{abstract}

Palavras-chave: qualidade de vida; otimismo; enfrentamento; estresse, câncer.

\begin{abstract}
Quality of life, optimism, coping, psychological morbidity and family stress in patients with colorectal cancer receiving chemotherapy. This study examined the relationships among quality of life, optimism, coping, psychological morbidity and family stress, the predictors of quality of life as well as differences in these variables according to gender, type and duration of treatment, age and education level in patients with colorectal cancer. A sample of 100 patients undergoing chemotherapy participated in this study. Results showed that women, patients diagnosed in the past twelve months and those who underwent surgery/chemotherapy presented more psychological morbidity. Psychological morbidity was found to be a predictor of quality of life. Age and level of education showed to be relevant particularly regarding the use of coping strategies and quality of life.The results emphasize the need for interventions to focus on morbidity, optimism and take into account gender, duration of diagnosis and type of treatment in particular.
\end{abstract}

Keywords: quality of life; optimism; coping; stress; cancer.

\section{Resumen}

Calidad de vida, optimismo, afrontamiento, estrés psicológico y morbilidad en pacientes con cáncer colorrectal en quimioterapia, Este estudio evaluó la relación entre la calidad de vida, el optimismo, el afrontamiento, la morbilidad psicológica, estrés familiar, los predictores de la calidad de vida y há analizado las diferencias en estas variables por sexo, el tipo y la duración del tratamiento, la edad y nivel de educación en pacientes con cáncer colorrectal. Una muestra de 100 pacientes a recibir quimioterapia participaron en el estudio. Los resultados revelaron que las mujeres, los pacientes diagnosticados en los últimos 12 meses y los que se sometió a cirugía/quimioterapia están en mayor riesgo de desarrollar morbilidad psicológica. La depresión demostró ser un predictor de la calidad de vida. La edad y la educación demostraron ser especialmente relevante en términos de estrategias de afrontamiento utilizadas y calidad de vida. Los resultados ponen de relieve la necesidad de intervenciones centrándose morbilidad, el optimismo y tienen en cuenta el género, la duración del diagnóstico y el tipo de tratamiento, en particular.

Palabras clave: calidad de vida; optimismo; afrontamiento; estrés, cáncer. 
O câncer colorretal constitui a segunda principal causa de morte por neoplasia no sexo feminino (570000 casos, $9,4 \%$ ) e a terceira no sexo masculino (663000 casos, $10 \%$ ), a nível mundial (Ferlay \& Shin, 2010). Em Portugal, de acordo com o Registo Nacional de Oncologia, a incidência do câncer colorretal é de 31,82/100,000 para as mulheres e 56,85/100,000 para os homens (Pontes, 2005).

A cirurgia e a quimioterapia são os tratamentos de escolha no câncer colorretal (Anjos \& Zago, 2006). Do procedimento cirúrgico pode surgir um estoma para a saída de fezes advindas do intestino grosso (cólon), podendo ser temporário ou definitivo (Castro, Scorza, \& Chem, 2011). De acordo com Camilleri-Brennan e Steele (2002), a presença de um estoma leva ao surgimento de alterações ao nível da imagem corporal, autoestima, e pode resultar em depressão, a qual pode dificultar as relações familiares (Castro et al., 2011), podendo contribuir para o surgimento de estresse familiar e, consequentemente para a alteração da qualidade de vida (Michelone \& Santos, 2004). Mahjoubi, Mirzaei, Azizi, Jafarinia e Zahedi-Shoolami (2012) acrescentam que a existência de um estoma está associado a um maior comprometimento da qualidade de vida destes pacientes, ao nível do funcionamento emocional.

O tratamento com quimioterapia contribui para o surgimento de efeitos colaterais ou toxicidades, sendo que em algumas pesquisas verificou-se o aumento dos sintomas de fadiga, insônias, dor, náuseas, vômitos (Maisey et al., 2002; Roque \& Forones, 2006) e toxicidades gastrointestinais (Roque \& Forones, 2006).

No estudo de Chaves e Gorini (2011), os resultados relativos à qualidade de vida dos pacientes com câncer colorretal em quimioterapia demonstraram que o domínio psicológico e o das relações sociais foram os mais afetados. Também observaram que à medida que os pacientes se aproximavam do fim do tratamento percebiam a sua qualidade de vida como sendo mais positiva (Chaves \& Gorini, 2011). Pereira, Cesarino, Martins, Pinto e Netinho, (2012) realizaram um estudo em paciente com câncer colorretal e concluíram que os domínios mais afetados da qualidade de vida foram o físico, psicológico e o social e, pertencer ao sexo feminino, ter baixos rendimentos, não ter parceiro sexual e ter falta de orientação são os fatores sociodemográficos e clínicos que estão relacionados com as diferenças na qualidade de vida. Porém, os pacientes apresentaram uma qualidade de vida satisfatória apesar de viverem com estoma intestinal irreversível (Pereira et al., 2012).

Gray et al. (2011) realizaram um estudo em que concluíram que os fatores fadiga e a depressão eram os que mais afetavam a qualidade de vida dos pacientes com câncer colorretal. Couvreur (2001) acrescenta que a dor é um sintoma que perturba a qualidade de vida destes pacientes, contribuindo para diminuição das relações sociais, alterações psicológicas e o surgimento de estresse a nível familiar.

De fato, a literatura (Pereira \& Roncon, 2010; Turagabeci, Nakamura, Kizuki, \& Takano, 2007) revela que o estresse familiar encontra-se inversamente relacionado com a qualidade de vida e de forma direta com a morbidade psicológica, ou seja, quanto maior o estresse familiar, maior a morbidade psicológica e menor a qualidade de vida.

O diagnóstico de câncer ocorre dentro de um sistema familiar, que é afetado em maior ou menor grau por este evento (Salci \& Marcon, 2010). Todas as alterações e consequentes reajustamentos advindos do diagnóstico de câncer podem contribuir para que a família os perceba como uma fonte de estresse familiar (Alves et al., 2007). Segundo Pereira e Lopes (2005), o estresse familiar surge quando não existe um equilíbrio entre as consequências da doença e os recursos que a família possui. São poucos os estudos que enfatizam o estresse familiar em pacientes com câncer. Porém, no estudo realizado por Casmarrinha (2008), verificou-se que os familiares dos doentes oncológicos, em fim de vida, tanto possuíam sentimentos de perda eminente como valorizavam a vida e os últimos momentos junto do familiar. Esta situação pode acarretar alterações psicológicas, dado a incerteza relativamente à morte do familiar que pode contribuir para o surgimento de estresse na família

Baider, Perez e De-Nour (1989) estudaram o ajustamento dos casais em que um dos parceiros possui câncer do cólon e verificaram que os pacientes do sexo masculino e os parceiros do sexo feminino adaptavam-se melhor à doença. Esta divergência entre ajustes e reajustes do casal, demais membros da família pode contribuir para o surgimento de estresse familiar e, consequentemente, para diminuição da qualidade de vida do doente (Pereira \& Roncon, 2010).

Hacpille (2000) partilha a opinião de que os doentes de câncer apresentam alterações a nível psicológico sendo as mais frequentes, a ansiedade e a depressão. O estudo de Pereira e Figueiredo (2008) concluiu que os doentes com câncer colorretal que realizaram cirurgia/quimioterapia e cirurgia/ radioterapia apresentavam níveis de depressão e ansiedade mais elevados, quando comparados com os que realizaram apenas a cirurgia. Para além disso, os que possuíam uma ostomia apresentavam níveis mais elevados de ansiedade e depressão em comparação com os demais.

No estudo de Hyphantis, Oshima e Forones (2011) verificou-se que os sintomas de ansiedade e depressão aumentaram de forma significativa nos pacientes com câncer colorretal, sendo que os homens apresentaram maior risco de desenvolver sintomatologia depressiva, e as diferentes dimensões da qualidade de vida diminuíram de forma significativa no período de um ano. Também se verificou que o distresse psicológico era um forte preditor da qualidade de vida (Hyphantis et al., 2011). O estudo realizado por Pereira, Figueiredo e Fincham (2011) verificou que a ansiedade e a depressão foram os principais preditores da qualidade de vida em doentes com câncer colorretal. Por sua vez, Alacacioglu et al. (2010) verificaram que a morbidade psicológica estava correlacionada com a qualidade de vida nos doentes com câncer colorretal. No estudo de Cotrim e Pereira (2008), a morbidade psicológica contribuiu para a diminuição da qualidade de vida, nestes pacientes. Tsunoda et al. (2005) verificaram que a depressão possui maior impacto na qualidade de vida global do que a ansiedade nos doentes com câncer colorretal.

Estudos têm ressaltado a importância da religião como estratégia de enfrentamento utilizada por doentes para enfrentarem situações adversas, como um diagnóstico de câncer, aliando-as ao suporte emocional, instrumental e informativo (Liberato \& Macieira, 2008; Panzini \& Bandeira, 2007). Deste modo, no estudo de Murken, Namini, Gross e Korber (2010), verificou-se que os homens utilizavam mais o pensamento positivo e apresentavam estratégias de enfrentamento menos ativas e que as mulheres usavam mais o enfrentamen- 
to religioso do que os homens. Para além disso, constatou-se uma forte relação entre o uso do enfrentamento religioso e uma maior adaptação das mulheres à doença quando comparadas com os homens (Murken et al., 2010).

Segundo McCaul et al. (1999), o enfrentamento a que os indivíduos recorrem para enfrentarem situações estressantes, como um diagnóstico de câncer, pode influenciar a qualidade de vida. Balboni et al. (2007) referem que muitos estudos realizados com pacientes oncológicos têm demonstrado que a religião relaciona-se de forma positiva com a qualidade de vida. Por sua vez, Gonçalves (2006) estudou o tipo de enfrentamento utilizado por sobreviventes de câncer do cólon e do reto e concluiu que os doentes utilizavam predominantemente as estratégias de enfrentamento ativo, nomeadamente cognitivas e comportamentais.

Mattews e Cook (2009) verificaram que o otimismo constitui uma dimensão importante em pessoas com câncer, dado os estudos enfatizarem o papel do otimismo na adaptação à doença. Sendo assim, Friedman et al. (1992) verificaram que o otimismo disposicional relacionava-se de forma positiva com enfrentamento comportamental e ativo. Por outro lado, o enfrentamento de evitamento encontrava-se negativamente relacionado com o otimismo disposicional. No estudo de Mazanec, Daly e Douglas (2010), verificou-se que o otimismo disposicional não foi considerado um fator primordial para a qualidade de vida em pacientes com câncer, pelo menos no momento inicial do diagnóstico e tratamento. Por outro lado, há estudos que comprovam a existência de uma influência positiva do otimismo na qualidade de vida de pacientes com câncer colorretal nomeadamente, ao nível do bem-estar, emocional e funcional dos doentes (Steginga, Lynch, Hawkes, Dunn, \& Aitken, 2009).

Hodges e Winstanley (2012) verificaram que o otimismo exercia um efeito positivo direto em pacientes sobreviventes de câncer (Hodges \& Winstanley, 2012). Sulkers et al. (2012) estudou os níveis de otimismo e pessimismo em pacientes com câncer comparando-os com um grupo controle saudável. Verificaram que apesar de os pacientes com câncer não serem mais otimistas do que o grupo controle, eram significativamente menos pessimistas (Sulkers et al., 2012). Gustavsson-Lillius, Julkunen, Keskivaara, Lipsanen e Hietanen (2012) estudaram também as relações entre o otimismo e a morbidade (depressão e ansiedade) em pacientes com câncer e verificaram que os pacientes mais otimistas mencionaram menos sintomas de ansiedade e depressão quando comparados com os indivíduos menos otimistas.

Tendo em consideração o Modelo Estresse-Enfrentamento na Doença Crónica (Maes, Leventhal \& De Ridder,1996), as características da doença e os fatores sociodemográficos juntamente com a resposta emocional e cognitiva à doença são fatores que influenciam o enfrentamento que é um preditor da qualidade de vida. No presente estudo, foi estudada a resposta emocional à doença através da morbidade psicológica (ansiedade e depressão). Segundo o modelo, o enfrentamento é influenciado por recursos externos e internos. Ao nível dos recursos externos, no presente estudo foi avaliado o estresse familiar e, ao nível dos recursos internos, o otimismo. As variáveis clínicas estudadas foram a presença de estoma, tipo de tratamento e duração do diagnóstico e, ao nível das variáveis sociodemográficas, a idade, sexo e o grau de escolaridade.
Neste sentido, o presente estudo teve por objetivo analisar a relação entre as variáveis estresse familiar, morbidade psicológica, otimismo, enfrentamento e qualidade de vida; analisar o impacto da presença/ausência de estoma, duração do diagnóstico e do tipo de tratamento (cirurgia/quimioterapia e quimioterapia), nas variáveis psicológicas referidas, bem como conhecer os melhores preditores da qualidade de vida.

\section{Método}

\section{Amostra}

A amostra deste estudo foi constituída por 100 pacientes, 44 mulheres e 56 homens, com câncer colorretal a realizar quimioterapia, com média de idade de 62 anos $(D P=$ 11 ), sendo que $55 \%$ possuía baixa escolaridade (ensino fundamental incompleto - até $4^{\mathrm{a}}$ série), $21 \%$ possuía escolaridade intermédia (ensino fundamental - da $5^{\mathrm{a}}$ à $8^{\mathrm{a}}$ série) e $24 \%$ possuía escolaridade alta (ensino médio ou $2^{\circ}$ grau). A maior parte dos participantes (67\%) tinha sido diagnosticada há menos de um ano e realizada cirurgia (87\%). Da amostra total, $20 \%$ dos doentes possuía um estoma. A Tabela 1 apresenta os resultados descritivos das variáveis psicológicas. A presente amostra apresenta uma baixa morbilidade psicológica e boa qualidade de vida tendo em conta os resultados normativos em doentes crônicos (Pais Ribeiro, Silva, Ferreira, Meneses, \& Baltar, 2007), existência de estresse familiar (Hudson, 1993), bom nível de otimismo (Pais Ribeiro, Pinto, \& Santos, 2008) e boas estratégias de enfrentamento tendo em conta os valores mínimo e máximos das subescalas, embora os autores não tenham reportado as médias obtidas na adaptação Portuguesa do Brief Cope.

\section{Instrumentos}

Questionário de enfrentamento- versão reduzida (Brief COPE) (Carver, 1997). Este questionário, de 28 itens, mede 14 estilos e estratégias de enfrentamento. Os alfas das escalas variaram entre 0,50 e 0,90. A versão adaptada de Pais Ribeiro e Rodrigues (2004) apresentou as mesmas propriedades psicométricas que a versão original (Carver, 1997), sendo que os alfas variaram entre 0,55 e 0,84 . No presente estudo, os alfas das escalas variaram entre 0,50 e 0,94 , tendo sido utilizadas as escalas de enfrentamento de religião, enfrentamento ativo e enfrentamento de suporte social emocional cujos alfas se encontravam acima de 0,70.

Questionário da qualidade de vida da organização europeia para a pesquisa e tratamento do câncer (EORTC QLQ-30) (Aaronson et al., 1993). Este instrumento, de 30 itens, avalia diversas dimensões da qualidade de vida através de cinco escalas de funcionamento (físico, de papéis, cognitivo, emocional e social), três de sintomas (fadiga, dor, e nâuseas/vômitos), uma de saúde/QV global e vários itens que avaliam diversos sintomas como perda de apetite, dispneia, problemas no sono, obstipação e diarreia (Aaronson et al., 1993). Um resultado nas escalas de funcionamento e na de saúde/ qualidade de vida geral representa um elevado nível de funcionamento e qualidade de vida. Um resultado nas escalas de sintomas representa um elevado nível de sintomatolo- 
gia. Os alfas das escalas variaram entre 0,54 e 0,86 . A versão adaptada de Pais Ribeiro et al. (2008) apresentou as mesmas propriedades da versão original, sendo que os alfas variaram entre 0,57 e 0,88 . No presente estudo, os alfas das escalas variaram entre 0,53 e 0,89 , tendo sido utilizadas nos testes de hipóteses as escalas funcionamento físico, social, emocional e cognitivo, saúde/QV global, fadiga e dor, cujos alfas encontravam-se acima de 0,60.

Índice de Relacionamento Familiar (IFR) (Hudson, 1993). O instrumento é constituído por 25 itens. Valores elevados indicam níveis elevados de estresse familiar. O alfa de Cronbach foi de 0,95. No presente estudo utilizou-se o questionário adaptado por Pereira e Roncon (2010) cujo alfa na adaptação foi 0,95 , revelando propriedades adequadas para avaliar as relações familiares. Acima de 30 significa existência de estresse familiar e acima de 70 estresse severo. No presente estudo, o alfa encontrado foi também de 0,95.

Teste de Orientação de Vida - Revisto (LOT-R) (Scheier, Carver, \& Bridges, 1994). O instrumento é constituído por 10 itens, quanto maior a pontuação, maior o otimismo dispo- sicional. O valor do alfa foi de 0,78 . A versão portuguesa de Laranjeira (2008) possui as mesmas propriedades do instrumento original (Scheier et al., 1994), apresentando um alfa de 0,71. Neste estudo, o alfa de Cronbach encontrado foi de 0,82.

Escala de Ansiedade e Depressão Hospitalar (HADS) (Zigmond \& Snaith,1983). O HADS é constituído por duas subescalas, ansiedade e depressão, cada uma com sete itens. Maior pontuação corresponde a maior morbidade psicológica. O ponto de corte para possibilidade de comportamento perturbado é 8 em cada uma das subescalas sendo os valores inferiores entre 8-10 indicativos de um possível comportamento clinico indicativo de perturbação e entre 11 e 21 de um provável comprometimento clinico. A versão portuguesa de McIntyre, Pereira, Soares, Gouveia e Silva (1999) possui as mesmas propriedades do instrumento original, apresentando um alfa de 0,94 na escala total, 0,91 na subescala ansiedade e de 0,88 para a depressão. Neste estudo o alfa da escala total foi de 0,87 , na subescala ansiedade 0,84 e de 0,73 na subescala da depressão.

Tabela 1

Estatísticas Descritivas das Variáveis Psicológicas

\begin{tabular}{lcccc}
\hline \multicolumn{1}{c}{ Variáveis } & Min & Max & Média & DP \\
\hline Depressão & 0 & 21 & 4,53 & 3,66 \\
Ansiedade & 0 & 21 & 6,22 & 4,67 \\
QLQ F. F. & 0 & 200 & 67,3 & 30,48 \\
QLQ F. S. & 0 & 80 & 116,4 & 17,75 \\
QLQ F. E. & 0 & 160 & 69,3 & 38,21 \\
QLQ F. C. & 0 & 80 & 93,0 & 14,23 \\
Saúde/Q.V Global & 2 & 140 & 66,0 & 25,72 \\
Fadiga & 0 & 120 & 34,0 & 26,48 \\
Dor & 0 & 80 & 16,43 & 18,80 \\
LOT-R & 0 & 24 & 30,52 & 5,950 \\
IFR & 0 & 100 & 4,91 & 10,86 \\
COPE Aceitação & 0 & 6 & 3,77 & 1,53 \\
COPE Ativo & 0 & 6 & 3,63 & 2,02 \\
COPE U.S.S.E. & 0 & 6 & 4,08 & 2,33 \\
COPE Religião & 0 & 6 & & 2,06 \\
\hline
\end{tabular}

\section{Procedimentos}

O estudo foi aprovado pela Comissão de Ética de dois hospitais portugueses, tendo sido autorizada a coleta de dados no serviço de oncologia desses hospitais. Os pacientes foram identificados pelas enfermeiras do serviço de oncologia quando da realização do tratamento de quimioterapia. Posteriormente, os pacientes foram convidados pelas investigadoras a participarem no estudo após terem sido explicados os objetivos e de ter sido garantida a confidencialidade dos dados recolhidos. Tratou-se de uma amostra de conveniência. Tendo em conta a realização dos testes estatísticos para um nível de significância de $5 \%$, poder de $80 \%$, e número de variáveis, foi estimado que uma amostra de 97 participantes era suficiente para a realização das análises mais exigentes da regressão múltipla (Cohen, Cohen, West, \& Aiken, 2003).
A percentagem de pacientes que recusaram participar foi de $5 \%(n=5)$. A aplicação dos instrumentos foi ministrada nos pacientes com baixa escolaridade. O preenchimento dos instrumentos levou em média 45 minutos. Os instrumentos foram aplicados segundo a ordem que foram acima mencionados. Os critérios de inclusão foram: (1) estar a realizar tratamento de quimioterapia; (2) ter idade superior a 18 anos; (3) ser capaz de responder aos questionários por escrito ou em forma de entrevista. Critérios de exclusão incluíram (1) não estar realizando tratamento de quimioterapia; (2) perturbações psicológicas ou psiquiátricas graveis (ex. processo demencial, esquizofrenia, psicoses). 


\section{Análise das medidas}

As relações entre as variáveis psicológicas foram avaliadas através do coeficiente de Correlação de Pearson. Os preditores da qualidade de vida geral foram avaliados através de duas regressões lineares múltiplas (método Enter), tendo em conta o tamanho da amostra. Na primeira foram introduzidas as variáveis sociodemográficas/clinicas e, na segunda, as variáveis psicológicas que se relacionaram com as subescalas da qualidade de vida. Para analisar as diferenças nas variáveis psicológicas e clínicas em função do sexo, foram utilizados três testes Manova para as subescalas (qualidade de vida, morbidade psicológica, enfrentamento) e um teste $t$ para os totais das escalas. Para testar as diferenças na qualidade de vida geral, otimismo, morbidade psicológica, estresse familiar e o enfrentamento em função do tipo de tratamento, foi utilizado um teste de Mann-Whitney, por não estarem cumprindo os pressupostos subjacentes à realização de testes paramétricos.

Tabela 2

Coeficiente de correlação de Pearson entre as Variáveis Psicológicas $(n=100)$

\section{Resultados}

\section{Relação entre otimismo, enfrentamento, qualidade de vida, morbidade psicológica e estresse familiar}

Os resultados da correlação de Pearson mostraram uma relação positiva significativa entre o otimismo e a qualidade de vida $r=0,332, p=0,001$, o enfrentamento ativo $r=0,217$, $p=0,030$ e uma relação negativa com a morbidade psicológica $r=-0,491, p<0,001$. O enfrentamento de suporte social emocional relacionou-se positivamente com a depressão $r=$ $0,219, p=0,028$ e negativamente com a qualidade de vida, ao nível do funcionamento físico $r=-0,321, p=0,001$. O enfrentamento religioso relacionou-se negativamente com a qualidade de vida, subescala funcionamento físico $r=-0,219, p=$ 0,028 . A qualidade de vida relacionou-se de forma negativa com a morbidade psicológica $r=-0,424, p \leq 0,001$. A morbidade psicológica relacionou-se positivamente com o estresse familiar $r=0,23, p=0,019$ (Tabela 2).

\begin{tabular}{|c|c|c|c|c|c|c|c|c|c|c|c|c|c|c|}
\hline Variáveis & 1 & 2 & 3 & 4 & 5 & 6 & 7 & 8 & 9 & 10 & 11 & 12 & 13 & 14 \\
\hline 1. FF & ----- & & & & & & & & & & & & & \\
\hline 2. FE & $0,34^{* * *}$ & ----- & & & & & & & & & & & & \\
\hline 3. FS & $0,45^{* * *}$ & $0,52^{* * *}$ & ----- & & & & & & & & & & & \\
\hline 4. FC & $0,29^{* *}$ & $0,44^{* * *}$ & $0,43^{* * *}$ & ----- & & & & & & & & & & \\
\hline 5. S/QVG & $0,28^{* *}$ & $0,49^{* * *}$ & $0,47^{* * *}$ & $0,26^{*}$ & ----- & & & & & & & & & \\
\hline 6. Fadiga & $-0,45^{* * *}$ & $-0,47^{* * *}$ & $-0,48^{* * *}$ & $-0,45^{* * *}$ & $-0,46^{* * *}$ & ----- & & & & & & & & \\
\hline 7. Dor & $-0,30^{* *}$ & $-0,13$ & $-0,30^{* *}$ & $-0,11$ & $-0,32^{* *}$ & $0,54^{* * *}$ & ---- & & & & & & & \\
\hline $\begin{array}{l}\text { 8. C. } \\
\text { Ativo }\end{array}$ & $-0,05$ & 0,03 & 0,06 & 0,05 & 0,14 & $-0,13$ & $-0,10$ & ----- & & & & & & \\
\hline C.S.S.E & $-0,32^{* *}$ & $-0,11$ & $-0,06$ & $-0,13$ & 0,05 & 0,09 & 0,09 & $0,31^{* *}$ & ----- & & & & & \\
\hline 10. C. R. & $-0,22^{*}$ & $-0,11$ & 0,03 & $-0,02$ & $-0,01$ & 0,12 & 0,09 & 0,17 & $0,22^{*}$ & ----- & & & & \\
\hline $\begin{array}{c}11 . \\
\text { LOT-R }\end{array}$ & $0,36^{* * *}$ & $0,41^{* * *}$ & $0,31^{* *}$ & $0,23^{*}$ & $0,33^{* *}$ & 0,02 & 0,04 & $0,22^{*}$ & $-0,05$ & 0,13 & ----- & & & \\
\hline $\begin{array}{l}12 . \\
\text { HADS }\end{array}$ & $-0,33^{* *}$ & $-0,65^{* * *}$ & $-0,56^{* * *}$ & $-0,50^{* * *}$ & $-0,42^{* * *}$ & $0,42^{* * *}$ & $-0,49^{* * *}$ & $-0,07$ & 0,14 & $-0,00$ & $-0,49^{* * *}$ & ----- & & \\
\hline $\begin{array}{l}13 . \\
\text { HADS-A }\end{array}$ & $-0,20^{*}$ & $-0,59^{* * *}$ & $-0,49^{* * *}$ & $-0,44^{* * *}$ & $-0,33^{* *}$ & $0,31^{* *}$ & $-0,40^{* * *}$ & $-0,03$ & 0,05 & $-0,02$ & $-0,40^{* * *}$ & $0,93^{* * *}$ & ----- & \\
\hline $\begin{array}{c}14 . \\
\text { HADS-D }\end{array}$ & $-0,43^{* * *}$ & $-0,60^{* * *}$ & $-0,53^{* * *}$ & $-0,47^{* * *}$ & $-0,46^{* * *}$ & $0,47^{* * *}$ & $-0,51^{* * *}$ & $-0,10$ & $0,22^{*}$ & 0,03 & $-0,51^{* * *}$ & $0,89^{* * *}$ & $0,66^{* * *}$ & ---- \\
\hline 15. IFR & 0,14 & $-0,06$ & $-0,08$ & 0,00 & $-0,14$ & 0,01 & 0,04 & 0,07 & $-0,09$ & $-0,11$ & $-0,02$ & $0,23^{*}$ & $0,34 * *$ & 0,05 \\
\hline
\end{tabular}

FF (funcionamento físico), FE (funcionamento emocional), FS (funcionamento social), FC (funcionamento cognitivo), S/QVG (saúde/qualidade de vida global), C.S.S.E (coping suporte social emocional), C.R. (coping religião), LOT-R (otimismo), HADS (morbilidade psicológica), HADS-A (ansiedade), HADS-D (depressão), IFR (stress familiar)

\section{Diferenças nas variáveis psicológicas e clínicas em função do sexo}

Os resultados da Manova revelaram diferenças significativas entre os gêneros ao nível do funcionamento social $[F$ $(1,98)=8,619, p=0,004]$, saúde e qualidade de vida global $[F(1,98)=5,466, p=0,021]$, fadiga $[F(1,98)=11,635, p=$ $0,001]$, e dor $[F(1,98)=4,472, p=0,037]$; do enfrentamento religião $[F(1,98)=4,538, p=0,036]$, e da morbidade psicoló- gica $[t(98)=2,736, p=0,007]$, e depressão $[F(1,98)=10,266$, $p=0,002]$. As mulheres possuem mais morbidade psicológica, depressão, fadiga e dor e utilizam mais o enfrentamento religioso. Os homens, por outro lado, possuem melhor funcionamento social e revelaram melhor qualidade de vida. Não foram encontradas diferenças significativas ao nível das restantes variáveis. 


\section{Diferenças nas variáveis psicológicas em função do tratamento}

Os resultados do teste Mann-Whitney revelaram diferenças significativas entre os pacientes que foram submetidos a tratamentos de quimioterapia/cirurgia e os que só foram submetidos a tratamento de quimioterapia ao nível do estresse familiar $[U=355,5, p=0,029]$. Assim, os pacientes que realizaram quimioterapia/cirurgia possuem maior estresse familiar em comparação com os que só receberam quimioterapia. Não se verificaram diferenças ao nível das restantes variáveis.

\section{Diferenças nas variáveis psicológicas em função da duração do diagnóstico}

Os resultados da Manova mostraram diferenças significativas ao nível do funcionamento físico $[F(1,98)=6,707, p=0,11]$. Os pacientes diagnosticados há menos de um ano possuem maior funcionamento físico em comparação com os restantes.

\section{Diferenças nas variáveis psicológicas em função da idade}

Os resultados do teste Mann-Whitney revelaram diferenças significativas em função da idade ao nível das subescalas funcionamento emocional [ $U=386,5, p=.031]$ e funcionamento social [ $U=373, p=0,013$ ]; e do enfrentamento suporte social emocional [ $U=406.5, p=0,044]$. Os pacientes com câncer colorretal com mais de 50 anos de idade possuem melhor funcionamento emocional e social e utilizam mais o suporte social emocional como estratégia de enfrentamento para lidarem com a doença. Não foram encontradas diferenças significativas nas restantes variáveis.

\section{Diferenças nas variáveis psicológicas} em função da escolaridade

Os resultados da Manova mostraram diferenças significativas em função da escolaridade ao nível da subescala funcionamento físico $[F(1,98)=4,076, p=0,046]$, do enfrentamento suporte social emocional $[F(1,98)=7,789, p$ $=0,006]$, do enfrentamento religião $[F(1,98)=15,285, p<$ $0,001]$ e do estresse intrafamiliar $[t(77,439)=-2.156, p=$ 0,034]. Sendo assim, os pacientes com baixa escolaridade utilizaram mais o suporte social emocional e a religião como estratégias de enfrentamento para lidarem com a doença, em comparação com os pacientes com maior escolaridade. Estes, por sua vez, apresentaram melhor funcionamento físico e mais estresse intrafamiliar. Não foram encontradas diferenças significativas ao nível das restantes variáveis.

\section{Preditores da qualidade de vida geral}

Os resultados da regressão hierárquica revelaram que apenas a depressão previu negativamente a qualidade de vida dos pacientes. Quanto maior a sintomatologia depressiva apresentada pelos pacientes, pior é a qualidade de vida geral. O modelo explicou $24,6 \%$ da variância, sendo que este percentual é considerado baixo. Nenhuma variável clinica ou socio demográfica se revelou como preditora da qualidade de vida geral (Tabelas 3 e 4).

Tabela 3

Modelo Final dos Preditores Sociodemográficos e Clínicos da Qualidade de Vida Geral (N=100)

\begin{tabular}{lcccc}
\hline Variáveis & \multicolumn{3}{c}{ Qualidade de Vida Geral } \\
& $\boldsymbol{R}^{\mathbf{2}}\left(\boldsymbol{R}^{\mathbf{2}} \mathbf{A d j}\right)$ & $\boldsymbol{F ( 5 , 9 4 )}$ & $\boldsymbol{\beta}$ & \multicolumn{1}{c}{$\boldsymbol{t}$} \\
\hline Sexo & & & 0,228 & 0,274 \\
Idade & & & 0,071 & $-0,660$ \\
Escolaridade & $0,302(0,091)$ & 0,103 & $-0,084$ & $-1,455$ \\
Duração do Diagnóstico & & & $-0,144$ & $-0,453$ \\
Tipo de Tratamento & & $-0,045$ & \\
\hline
\end{tabular}

Tabela 4

Modelo Final dos Preditores Psicológicos da Qualidade de Vida Geral (N=100)

\begin{tabular}{|c|c|c|c|c|}
\hline \multirow{2}{*}{ Variáveis } & \multicolumn{4}{|c|}{ Qualidade de Vida Geral } \\
\hline & $R^{2}\left(R^{2} A d j\right)$ & $F(5,94)$ & $\boldsymbol{\beta}$ & $\boldsymbol{t}$ \\
\hline Enfrentamento Suporte Social Emocional & & & $-0,409$ & $-3,109$ \\
\hline Enfrentamento Religioso & & & $-0,019$ & $-0,158$ \\
\hline Otimismo & $0,496(0,246)$ & $6,140^{* * *}$ & 0,133 & 1,158 \\
\hline Depressão & & & $-0,056$ & $-0,599^{*}$ \\
\hline Ansiedade & & & 0,164 & 1,728 \\
\hline
\end{tabular}

${ }^{*} p \leq 0,05 ;{ }^{* * *} p \leq 0,001$ 


\section{Discussão}

O presente estudo mostrou que os pacientes mais otimistas revelaram ter melhor qualidade de vida e utilizaram mais o enfrentamento ativo quando comparados com os pacientes menos otimistas, dada a relação positiva entre a variável otimismo e a qualidade de vida e enfrentamento ativo. Os pacientes menos otimistas, por sua vez, possuíram mais morbidade psicológica, ansiedade e depressão. De fato, há estudos que revelam a existência de uma influência positiva do otimismo na qualidade de vida de pacientes com câncer colorretal (Steginga et al., 2009) e que o otimismo disposicional se relacionava de forma positiva com o enfrentamento ativo (Friedman et al., 1992). Gustavsson-Lillius et al. (2012) referem que os pacientes otimistas possuem menos sintomatologia psicológica do que os menos otimistas.

Os pacientes que mais utilizavam a religião como estratégia de enfrentamento perante a doença possuíam menor qualidade de vida ao nível do funcionamento físico, entendase como fazer esforços (i.e. carregar sacos), andar a pé, necessitar de ficar na cama ou numa cadeira, comer, vestir, lavar-se ou ir ao banheiro. Estes resultados divergem dos estudos realizados com pacientes oncológicos que revelam que a qualidade de vida e o bem-estar religioso se relacionam de forma positiva (Balboni et al., 2007). Uma explicação para os resultados pode estar no fato de esses pacientes, ao possuírem um menor funcionamento físico em termos de qualidade de vida, poderem recorrer a uma maior utilização da religião como estratégia de enfrentamento, por talvez entenderem que essa estratégia os possa ajudar na sua recuperação enquanto os pacientes com um melhor funcionamento físico poderão não sentir tanto essa necessidade. Por sua vez, os pacientes que percebiam a sua qualidade de vida como sendo boa possuíam menores níveis de morbidade psicológica, ansiedade e depressão do que os demais. Estes resultados vão ao encontro dos estudos de Alacacioglu et al. (2010), o qual refere que a morbidade psicológica encontra-se fortemente correlacionada com a qualidade de vida, e de Hyphantis et al. (2011), o qual revelou que sintomatologia depressiva e ansiosa aumentou enquanto a qualidade de vida diminuiu de forma significativa.

Os pacientes que apresentavam mais morbidade psicológica possuíam mais estresse familiar. Nos estudos de Turagabeci et al. (2007), Pereira e Roncon, (2010) verificou-se que o estresse familiar se relacionou de forma direta com a morbidade psicológica. Os estudos de Panzini e Bandeira (2007) e de Liberato e Macieira (2008) têm ressaltado a importância do suporte emocional como estratégia de enfrentamento que os doentes utilizam para enfrentarem situações adversas influenciando a sua qualidade de vida (McCaul et al., 1999). Assim, os resultados estão de acordo com a literatura na medida em que os pacientes que utilizavam mais o enfrentamento de suporte emocional possuíam pior funcionamento físico e maiores níveis de depressão.

Os resultados mostraram que as mulheres possuíam mais depressão, fadiga e dor e utilizavam mais o enfrentamento de religião do que os homens. Estes, por outro lado, possuíam maior funcionamento social e percebiam melhor a sua qualidade de vida em comparação com as mulheres. O estudo de Hyphantis et al. (2011) referiu que os homens com câncer co- lorretal apresentaram maior risco de desenvolver sintomatologia depressiva do que as mulheres, o que não corrobora os resultados do presente estudo. Porém, os resultados podem ser explicados pelo fato de as mulheres possuírem mais fadiga do que os homens e isso pode ter contribuído para a presença de maior sintomatologia depressiva, dado que no estudo de Santos, Mota e Pimenta (2009) a fadiga e a depressão encontraram-se relacionadas de forma positiva em pacientes com câncer colorretal. O estudo de Nicolussi e Sawada (2009) verificou que as mulheres com câncer colorretal apresentaram mais dor e fadiga em comparação com os homens e o estudo de Murken et al. (2010) revelou que as mulheres utilizavam mais enfrentamento religioso do que os homens, tal como se verificou no presente estudo. O estudo de Pérez, Navarro e de la Fuente (2004) mostrou que os homens possuíam melhor qualidade de vida em comparação com as mulheres, o que vai de encontro aos resultados encontrados.

Ao nível do tratamento, os pacientes que realizaram cirurgia/quimioterapia possuíam mais estresse familiar em comparação com os pacientes que só receberam quimioterapia. De fato o diagnóstico de câncer pode causar impacto não só nos pacientes como também nos seus familiares, nas diferentes fases da doença, nomeadamente durante o tratamento (Nicolussi \& Sawada, 2009). Este processo pode contribuir para o surgimento de efeitos adversos ao nível do relacionamento familiar (Arndt, Merx, Stegmaier, Ziegler, \& Brenner, 2004). A explicação para as diferenças encontradas entre os grupos pode residir no fato de os dois tratamentos em conjunto poderem provocar mais problemas e limitações do que isoladamente e consequentemente levar ao aumento do estresse familiar.

O nosso estudo mostrou que os pacientes diagnosticados há mais de um ano possuíam menor funcionamento físico quando comparados com os pacientes diagnosticados nos últimos doze meses. Hobday et al. (2002) verificou que os pacientes que concluíram o tratamento para o cancro colorretal apresentavam uma qualidade de vida global melhor, apesar de possuírem um funcionamento físico pior quando comparados com a avaliação inicial realizada antes do tratamento. Contudo, Chaves e Gorini (2011), verificaram que os pacientes diagnosticados há menos tempo demonstraram uma pior qualidade de vida quando comparados com os que se encontravam no final do tratamento.

Foi possível verificar no nosso estudo que os pacientes com cancro colorretal com mais de 50 anos de idade possuíam melhor funcionamento emocional e social e utilizavam mais o suporte social emocional como estratégias de enfrentamento para lidarem com a doença. Por outro lado, os pacientes com baixa escolaridade utilizavam mais o suporte social emocional e a religião como estratégias de enfrentamento para lidarem com a doença, em comparação com os pacientes com maior escolaridade que por sua vez apresentavam melhor funcionamento físico e mais estresse familiar. Poucos são os estudos que se têm debruçado sobre a avaliação da qualidade de vida, do enfrentamento e do estresse familiar nos pacientes com câncer colorretal em função da idade e escolaridade. Porém, Pereira et al. (2012) no seu estudo não encontrou diferenças na qualidade de vida nos pacientes com câncer colorretal em função da idade e da escolaridade. 
Relativamente ao uso do enfrentamento em função da idade, os resultados do nosso estudo podem ser explicados pelo fato de os pacientes com mais de 50 anos de idade ao possuírem um maior funcionamento social e emocional recorrerem mais a este tipo de suporte como estratégia de enfrentamento perante a doença, dado considerarem esta estratégia eficaz nesse momento da sua vida. Lopes (2007) refere uma pessoa com mais escolaridade, tem recursos para poder ultrapassar melhor uma mudança (e.g. diagnóstico de câncer) do que uma pessoa com baixo nível de escolaridade, sendo que esta tende a tornar-se mais dependente do seu suporte social. Sendo assim, compreende-se que o paciente com baixa escolaridade possa fazer mais uso desse suporte como estratégia de enfrentamento perante a doença.

A depressão previu a qualidade de vida global dos pacientes com câncer colorretal. Assim sendo, aqueles que apresentavam maiores níveis de depressão percebiam a sua qualidade de vida como sendo pior do que os demais embora a variância explicada tenha sido baixa provavelmente porque se tratam de uma amostra com baixa morbilidade psicológica e boa qualidade de vida geral. Contudo, os resultados vão ao encontro de outros estudos (Hyphantis, 2011; Pereira et al., 2011) que revelaram que a depressão foi um preditor da qualidade de vida dos pacientes com câncer colorretal.

\section{Limitações}

O fato de os participantes pertencerem apenas a dois hospitais do país não permite a generalização dos resultados e obriga a uma interpretação cautelosa. Outra limitação refere-se ao uso exclusivo de questionários de autorrelato e ao fato de não ter sido possível a utilização de algumas escalas do questionário de enfrentamento e do questionário da qualidade de vida, como dado o valor de alfa. Finalmente, a amostra apresenta baixa morbilidade psicológica e uma boa qualidade de vida geral pelo que seria importante replicar os resultados em amostras mais heterogêneas.

\section{Conclusões}

A relação positiva existente entre o otimismo e enfrentamento ativo e qualidade de vida, a relação negativa entre otimismo e morbidade psicológica, bem como a relação negativa entre depressão e qualidade de vida sugere que intervenções focadas no otimismo e na morbidade poderão contribuir para qualidade de vida em pacientes com câncer colorretal. Por sua vez, a intervenção deve ter em consideração o tipo de tratamento e o gênero.

Investigações futuras, com recurso a amostras maiores deveriam avaliar o papel do otimismo como moderador na relação entre a morbidade psicológica e a qualidade de vida e incluir variáveis como a imagem corporal avaliando a possibilidade do seu papel mediador entre o otimismo e a qualidade de vida.

\section{Referências}

Aaronson, N., Ahmedzai, S., Bergman, B., Bullinger, M., Cull, A., Duez, N J, ... Haes, J. C. (1993). The European organization for research and treatment of cancer QLQ-C30: A quality-of life-instrument for use in international clinical trials in oncology. Journal of the National Cancer Institute, 85(5), 365-376. doi: 10.1590/S0104-11692006000100005

Alacacioglu, A., Binicier, O., Gungor, O., Oztop, I., Dirioz, M., \& Yilmaz, U. (2010). Quality of life, anxiety and depression in Turkish colorectal cancer patients. Journal of Supportive Care Cancer, 18(4), 417-421. doi:10.1007/s00520-009-0679-2.25

Alves, L., Leimann, B. C. Q., Vasconcelos, M. E. L., Carvalho, M. S., Vasconcelos, A. G. G., Fonseca, T. C. O, ... Laurenti, R. (2007). A influência das doenças crónicas na capacidade funcional dos idosos do município de São Paulo, Brasil. Cadernos de Saúde Pública, 23(8), 1924-1930. doi: 10.1590/S0102-311X2007000800019

Anjos, A., \& Zago, M. (2006). A experiência da terapêutica quimioterápica oncológica na visão do paciente. Revista Latino-Americana de Enfermagem, 14(1), 33-40. doi: 10.1590/S0104-11692006000100005

Arndt, V., Merx, H., Stegmaier, C., Ziegler, H., \& Brenner, H. (2004). Quality of life in patients with colorectal cancer 1 year after diagnosis compared with the general population: A population-based study. Journal of Clinic Oncology, 22(23), 4829-36. doi: 10.1200/JCO.2004.02.018

Baider, L., Perez, T., \& Kaplan De-Nour, A. (1989). Gender and adjustment to chronic disease: A study of couples with colon cancer. General Hospital Psychiatry, 11(1), 1-8. doi: 10.1016/0163-8343(89)90018-2

Balboni, T. A., Vanderwerker, L. C., Block, S. D., Paulk, E. M., Lathan, C.S., Peteet, J. R., \& Prigeson, H. G. (2007). Religiousness and spiritual support among advanced cancer patients and associations with end-of-life treatment preferences and quality of life. Journal of Clinical Oncology, 25(5), 555-560. Recuperado de http://jco.ascopubs.org/content/25/5/555.full.pdf

Camilleri-Brennan, J., \& Steele, R. J. C. (2002). Objective assessment of morbidity and quality of life after surgery for low rectal cancer. Colorectal Disease, 4(1), 61-6. doi: 10.1046/j.1463-1318.2002.00300.x

Carver, C. S. (1997). "You want to measure coping but your protocol is too long: Consider the brief COPE.” International Journal of Behavioral Medicine, 4(1), 92-100. doi: 10.1207/s15327558ijbm0401_6

Casmarrinha, M. (2008). Familiares do doente oncológico em fim de vida dos sentimentos às necessidades (Dissertação de Mestrado). Universidade do Porto, Portugal. Recuperado de http://hdl.handle.net/10216/7169

Castro, E., Scorza, A., \& Chem, C. (2011). Qualidade de vida e indicadores de ansiedade e depressão de pacientes com cancer colorretal. Psicologia, Saúde e Doença, 12(1), 131-142. Recuperado de http://www.scielo.mec.pt/scielo.php?script=sci_arttext\&pid=S1645-00862011000100009\&lng=pt\&tlng=pt

Chaves, P., \& Gorini, M. (2011). Qualidade de vida do paciente com câncer colorretal em quimioterapia ambulatorial. Revista Gaúcha de Enfermagem 32(4), 767-773. doi: 10.1590/S1983-14472011000400018

Cohen, J., Cohen, P., West, S. G., \& Aiken, L. S. (2003). Applied multiple regression/correlation analysis for the behavioral sciences ( $3^{a}$ ed.). Mahwah, NJ: Lawrence Earlbaum Associates.

Couvreur, C. (2001). A Qualidade de vida: Arte para viver no século XXI. Loures: Lusociência.

Cotrim, H., \& Pereira, M. G. (2008). Impact of colorectal cancer on patient and family: Implications for care. European Journal of Oncology Nursing 12(3), 217-226. doi: 10.1016/j.ejon.2007.11.005

Ferlay, J., \& Shin, H. (2010). Estimates of worldwide burden of cancer in 2008 GLOBOCAN 2008. Journal Cancer, 127(12), 2893-2917. doi: 10.1002/ ijc. 25516

Friedman, L., Nelson, D., Baer, P., Lane, M., Smith, F., \& Dworkin, R. (1992) The relationship of dispositional optimism, daily life stress, and domestic environment to coping methods used by cancer patients. Journal of Behavioral Medicine, 15(2), 127-141. 10.1007/BF00848321 
Gonçalves, S. (2006). Para além da doença: sobreviventes de cancer do colon e reto satisfação sexual, autoeficácia e enfrentamento (Dissertação de Mestrado). Instituto Superior da Psicologia Aplicada, Portugal. Recuperado de http:// repositorio.ispa.pt/bitstream/10400.12/1259/1/DM\%20GONC-S1.pdf

Gray, N. M., Hall, S. J., Browne, S., Macleod, U., Mitchell, E., Lee, A. J.... Campbell, N. C. (2011). Modifiable and fixed factors predicting quality of life in people with colorectal cancer. British Journal of Cancer, 104, 16971703. doi:10.1038/bjc.2011.155

Gustavsson-Lillius, M., Julkunen, J., Keskivaara, P., Lipsanen, J., \& Hietanen, P. (2012). Predictors of distress in cancer patients and their partners: The role of optimism in the sense of coherence construct. Psychology \& Health, 27(2), 178-95. doi: 10.1080/08870446.2010.484064.

Hacpille, L. (2000). Abordagem em cuidados paliativos: a dor cancerosa e seu tratamento. Lisboa: Instituto Piaget.

Hobday, T. J., Kugler, J. W., Mhaoney, M. R., Sargent, D. J., Sloan, J. A., Fitch, T. R.... Golberg, R. M. (2002) Efficacy and quality-of-life data are related in a phase II trial of oral chemotherapy in previously untreated patients with metastatic colorectal carcinoma. Journal of Clinic Oncology, 20(23), 4574-80. doi: 10.1200/JCO.2002.08.535JCO

Hodges, K., \& Winstanley, S. (2012). Effects of optimism, social support, fighting spirit, cancer worry and internal health locus of control on positive affect in cancer survivors: A path analysis. Stress and Health, 28(5), 408-415. doi: 10.1002/smi.2471

Hudson, W. (1993). Index of family relations: The Walmyr assessment scales scoring manual. Tempe: Walmyr Publishing Company.

Hyphantis, T., Oshima, C., \& Forones, N. (2011). Personality variables as predictors of early non metastatic colorectal cancer patients' psychological distress and health-related quality of life: a one year prospective study. Journal of Psychomatic Research, 70(5), 411-421. doi: 10.1016/j. jpsychores

Laranjeira, C. (2008). Tradução e validação portuguesa do revised life orientation test (LOT-R). Universitas Psychologica, 7(2), 469-476. Recuperado http://revistas.javeriana.edu.co/sitio/psychologica/sccs/articulo. php?id=12

Liberato, R., \& Macieira, R. (2008). Espiritualidade no enfrentamento do câncer. In V. Carvalho, M. H. P. Franco, M. J. Kovács, R. P. Liberato, R. C. Macieira, M. T. Veit, M. J. B. Gomes, \& L. H. C. Barros (Orgs.), Temas em Psicooncologia (p. 414-431). São Paulo: Summus Editorial.

Longacre, A., Cramer, L., \& Gross, C. (2006). Screening colonoscopy use among individuals at higher colorectal cancer risk. Journal of Clinic Gastroenterology, 40, 490-6.

Lopes, A. (2007). Generalidades e singularidades da doença em família: perceção da qualidade de vida, estresse e enfrentamento (Dissertação de Mestrado). Faculdade de Psicologia e de Ciências da Educação da Universidade de Coimbra, Coimbra. Recuperado de http://www.psicologia.pt/teses/textos/TE0001.pdf

Maes, S., Leventhal, H., \& De Ridder, D. T. D. (1996). Coping with chronic disease. In M. Ziender \& N. S. Endler (Orgs.), Handbook of coping: Theory, research, applications (p. 221-245). Nova Iorque: John Wiley \& Sons, Inc.

Mahjoubi, B., Mirzaei, R., Azizi, R., Jafarinia, M., \& Zahedi-Shoolami, L. (2012). A cross-sectional survey of quality of life in colostomates: A report from Iran. Health and Quality of Life Outcomes, 10, 136. doi: 10.1186/1477-7525-10-136

Maisey, N. R., Norman, A., Watson, M., Allen, M. J., Hill, M. E., \& Cunningham, D. (2002) Baseline quality of life predicts survival in patients with advanced colorectal cancer. European Journal of Cancer, 38, 1351-7.

Mattews, E. E., \& Cook, P. F. (2009). Relationships among optimism, well-being, self-transcendence, coping, and social support in women during treatment for breast cancer. Psycho-Oncology, 18, 716-726. doi: 10.1002/pon.1461

Mazanec, S., Daly, B., \& Douglas, S. (2010). The relationship between optimism and quality of life in newly diagnosed cancer patients. Cancer Nursing, 33(3), 235-243. doi:10.1097/NCC.0b013e3181c7fa80
McCaul, K., Sandgren, A., King, B., O’Donnell, S., Branstetter, A., \& Foreman, G. (1999). Coping and adjustment to breast cancer. Psychooncology, 8, 230-236. doi: 10.1002/(SICI)1099-1611(199905/06)8:3<230::AIDPON374>3.0.CO;2-\#

McIntyre, M. T., Pereira, G., Soares, V., Gouveia, J., \& Silva, S. (1999). Escala de ansiedade e depressão hospitalar. Versão portuguesa de investigação. Braga, Portugal: Departamento de Psicologia, Universidade do Minho.

Michelone, A., \& Santos, V. (2004). Qualidade de vida de adultos com câncer colorretal com e sem colostomia. Revista Latino-Americana de Enfermagem, 12(6), 875-83. doi: 10.1590/S0104-11692004000600005

Murken, S., Namini, S., Gross, S., \& Korber, J. (2010). Gender specific differences in coping with colon cancer: Empirical findings with special consideration of religious coping. Die Rehabilitation, 49(2), 95-104. doi: $10.1055 / \mathrm{s}-0030-1249029$

Nicolussi, A., \& Sawada, N. (2010). Fatores que influenciam a qualidade de vida de pacientes com câncer de cólon e reto. Acta Paulista de Enfermagem, 23(1), 125-30. doi: 10.1590/S0103-21002010000100020

Pais Ribeiro, J., \& Rodrigues, A. P. (2004). Questões acerca do coping: a propó sito do estudo de adaptação do Brief COPE. Psicologia, Saúde e Doenças, 5(1), 3-15. Recuperado de http://www.scielo.mec.pt/scielo.php?scrip$\mathrm{t}=$ sci_arttext\&pid=S1645-00862004000100001\&lng=es\&tlng=pt

Pais Ribeiro, J., Pinto, C., \& Santos, C. (2008). Validation study of the Portuguese version of the QLC-C30-V.3. Psicologia, Saúde e Doenças, 9(1), 89102. Recuperado de http://www.redalyc.org/articulo.oa?id=36290108

Pais Ribeiro, J., Silva, I., Ferreira, T., Martins, A., Meneses, R., \& Baltar, M. (2007). Validation study of a Portuguese version of the hospital anxiety and depression scale. Psychology, Health and Medicine, 12(2), 225-35. doi: 10.1080/13548500500524088

Panzini, R., \& Bandeira, D. (2007). Coping (enfrentamento) religioso/espiritual. Revista de Psiquiatria Clínica, 34(suppl. 1), 126-135. Recuperado de http://www.hcnet.usp.br/ipq/revista/vol34/s1/pdf/126.pdf

Pereira, A., Cesarino, C., Martins, M., Pinto, M., \& Netinho, J. (2012). Associação dos fatores sociodemográficos e clínicos à qualidade de vida dos estomizados. Revista Latino-Americana Enfermagem, 20(1). doi: org/10.1590/S0104-11692012000100013

Pereira, M. G., \& Figueiredo, A. P. (2008). Depressão, ansiedade e stress póstraumático em doentes com cancro colorretal: Validação do hospital anxiety and depression scale (HADS) e Impacto of Events Scale (IES) numa amostra de doentes oncológicos. Oncology News, 5, 11-19. Recuperado de http://hdl.handle.net/1822/16463

Pereira, M. G., \& Roncon, J. (2010). Relacionamento familiar em pessoas idosas: adaptação do índice de relações familiares (IFR). Psicologia, Saúde e Doenças, 11(1), 41-53. Recuperado de http://www.scielo.mec.pt/scielo.php?script=sci_arttext\&pid=S1645-00862010000100004\&lng=pt\&tlng=pt

Pereira, M. G., Figueiredo, A. P., \& Fincham, F. D. (2011). Anxiety, depression, traumatic stress and quality of life in colorectal cancer after different treatments: A study with Portuguese patients and their partners. European Journal of Oncology Nursing, 16(3), 227-232. doi: 10.1016/j. ejon.2011.06.006

Pérez, L., Navarro Piñero, A., \& de La Fuente Perucho, A. (2004). Study of factors related to quality of life in patients with locally advanced rectal cancer. $R e$ vista Espanhola de Enfermagem, 96(11), 746-57. Recuperado de http://scielo. isciii.es/scielo.php?script=sci_arttext\&pid=S1130-01082004001100002

Pontes, L. (2005). Registo oncológico nacional. Portugal: Instituto Português de Oncologia do Porto. Recuperado de http://www.croc.min-saude.pt/ NR/rdonlyres/6E2B1673-B238-4FEC-B324-D20EC1AA4F9B/19444/ RONPublica\%C3\%A7\%C3\%A3onacional2005.pdf

Roque, V. M. N., \& Forones, N. M. (2006). Avaliação da qualidade de vida e toxicidades em pacientes com câncer colorretal tratados com quimioterapia adjuvante baseada em Fluoropirimidinas. Arquivos de Gastroenterologia, 42(2), 94-101. doi: org/10.1590/S0004-28032006000200007

Salci, M. A., \& Marcon, S. S. (2011). Enfrentamento do câncer em família. Texto Contexto de Enfermagem, 20, 178-186. doi: 10.1590/S010407072011000500023 
Santos, J., Mota, D., \& Pimenta, C. (2009). Co-morbidade fadiga e depressão em pacientes com câncer colorretal. Revista da Escola de Enfermagem USP, 43(4), 909-14. doi: 10.1590/S0080-62342009000400024

Scheier, M., Carver, C., \& Bridges, M. (1994). Distinguishing optimism from neuroticism (and Trait anxiety, Self-Mastery, and Self-Esteem): A Reevaluation of the life orientation test. Journal of Personality and Social Psychology, 67(6), 1063-1078. doi: 10.1037/0022-3514.67.6.1063

Steginga, S., Lynch, B., Hawkes, A., Dunn, J., \& Aitken, J. (2009). Antecedents of domain-specific quality of life after colorectal cancer. Psycho-Oncology, 18, 216-220. doi: 10.1002/pon.1388

Sulkers, E., Fleer, J., Brinksma, A., Roodbol, P. F., Kamps W. J., \& Sanderman, R. (2012). Dispositional optimism in adolescents with cancer: Differential associations of optimism and pessimism with positive and negative aspects of well-being. British Journal of Health Psychology, 18(3), 474489. doi: 10.1111/j.2044-8287.2012.02096.x

Tsunoda, A., Nakao, K., Hiratsuka, K., Yasuda, N., Shibusawa, M., \& Kusano, M. (2005). Anxiety, depression and quality of life in colorectal cancer patients. Japan Society of

Clinical Oncology, 10, 411-417. doi: 10.1007/s10147-005-0524-7

Turagabeci, A., Nakamura, K., Kizuki, M., \& Takano, T. (2007). Family structure and health, how companionship acts as a buffer against ill health. Health and Quality of Life Outcomes, 5, 61. doi: 10.1186/1477-7525-5-61

Zigmond, A. S., \& Snaith, R. P. (1983). The hospital and anxiety and depression scale. Acta Psiquiátrica Scandinavica, 7, 361-370. 
Ana Sofia Cordeiro Botelho, Mestre em Psicologia Clínica e da Saúde pela Universidade do Minho, Psicóloga Clínica e da Saúde no Centro de Saúde de Ponta Delgada, São Miguel, Açores. Endereço para correspondência: Universidade do Minho. Escola de Psicologia, Campus de Gualtar, 4710-057, Braga, Portugal.

E-mail: ana.botelho89@hotmail.com

Maria da Graça Pereira, Doutora em Psicologia Clínica pela Universidade do Minho, é Professora Associada na Escola de Psicologia - Departamento de Psicologia Aplicada, Universidade do Minho.

E-mail: gracep@psi.uminho.pt 